ИЗВЕСТИЯ АКАДЕМИИ НАУК ЭСТОНСКОИ ССР. ФИЗИКА * МАТЕМАТИКА PROCEEDINGS OF THE ACADEMY OF SCIENCES OF THE ESTONIAN SSR. PHYSICS * MATHEMATICS

$1984,33,3$

\title{
ПРОСТРАНСТВЕННО-ВРЕМЕННАЯ ГОЛОГРАФИЯ ИМПУЛЬСНЫХ СВЕТОВЫХ ПОЛЕЙ В ВЫСОКОСЕЛЕКТИВНЫХ ФОТОХРОМНЫХ СРЕДАХ
}

\author{
(Представил К. К. Ребане)
}

В традиционной голографии на светочувствительном материале фиксируется пространственная зависимость поля, рассеянного от предметаобъекта. При считывании информации с голограммы изображение объекта восстанавливается с условием, что при записи во время экспозиции в объеме голограммы существовало некоторое стационарное распределение интенсивности поля. Это требование обусловлено способностью фоточувствительной среды регистрировать лишь суммарную дозу светового облучения, в которой информация о временных зависимостях поля утеряна (усреднена).

В цикле работ $\left[{ }^{1-5}\right]$ показано, что на высокоселективных фотохромных материалах, т. е. в средах, способных запоминать спектральный состав поглощенного света, возможна и голография временных зависимостей поля. Эксперименты, проведенные на замороженных матрицах с фотохимически активными примесными молекулами, где были занисаны и с высокой эффективностью, $(\sim 50 \%)$ восстановлены пикосекундные световые сигналы, показывают перспективность применения подобных материалов.

В настоящей работе развита теория пространственно-временной голографии на высокоселективных фотохромных материалах как метода фиксирования объектной сцены с последующим восстановлением изображения сцены во всей ее временной зависимости. В качестве последней допускается не только движение объекта или его частей, но и возникновение-исчезновение самосветящихся источников, амплитудная и даже частотная модуляция их излучения и т. д. При использовании низкотемпературных примесных систем с неоднородным распределением частот переходов и поддающихся фотохимическому выжиганию провала в сгектре поглощения [6,7], максимальная длительность записываемых сцен может достигать десятков наносекунд, а временное разрешение десятков фсмтосекунд. Это вытекает из основной идеи метода $\left[{ }^{2}\right]$ : в каждом элементе среды фиксируется взаимный спектр объектного и опорного импульсов в данной точке; в дальнейшем для считывающего импульса каждый элемент служит спектральным фильтром, формирующим из считывающего импульса реплику объектного импульса. Взаимный спектр, или «спектральная голограмма», записывается путем уничтожения части поглощающих центров в пределах неоднородной ширины $\left(\sim 10^{2} \mathrm{~cm}^{-1}\right)$ полосы поглощения матрицы с точностью однородной ширины $\left(>10^{-4} \mathrm{~cm}^{-1}\right)$ (бесфононной) линии [[ $\left.{ }^{8}\right]$ поглощения центров. Об- 
ратные значения этих ширин и определяют вышеприведенные временные границы.

Восстановление объектного импульса из считывающего можно интерпретировать так же, как образование сигнала затухания свободной поляризации на элементе среды с приготовленным при записи специальным распределением частот диполей. В такой временной трактовке очевидно, что время фазовой релаксации $T_{2}$ резонансного перехода молекулярных диполей - обратная величина ширине линий - ограничивает возможную длительность восстановленного импульса. Кроме того, фазовая память среды позволяет при записи разнести объектный и опорный импульсы во времени без потери интерференции между ними, что, как мы увидим в дальнейшем, имеет такую же практическую ценность, как использование наклонного опорного пучка в обычной голографии. Из временной трактовки следует также, что процесс записи и восстановления голограмм в рассматриваемых средах основан на физическом явлении, аналогичном стимулированному фотонному эхо в обычных резонансных средах. В связи с этим в $\left[{ }^{2}\right]$ было введено название фотохимически аккумулированного стимулированного светового эхо (ФАССЭ).

Возможности светового эхо в динамической голографии основательно проанализированы в ряде работ (см., напр., $\left.\left[{ }^{9-11}\right]\right)$. В [12], в частности, показано наличие корреляций во временных профилях импульсов стимулированного эхо и накачки. В теоретической работе $\left[{ }^{13}\right]$, вышедшей во время постановки первых наших экспериментов, показано как пространственно-временное поведение импульса лазера может быть записано в спектрально неоднородной резонансной среде и восстановлена в виде сигнала свободной поляризации.

Однако практическая применимость светового эхо для целей голографии ограничена тем обстоятельством, что голограмма весьма недолговечна (ограничена временем жизни возбужденного состояния молекул $10^{-9}-1$ с) и требует также высокой мощности импульсов накачки ( $\sim 10^{6}$ Вт в пикосекундном диапазоне). В противоположность этому, основанные на ФАССЭ голограммы истинные в том смысле, что допускают неоднократное считывание по истечении нескольких часов, а может быть и лет (в зависимости от стабильности продуктов фотохимических реакций); кроме того, высокого контраста легко достигнуть произвольно слабыми импульсами, многократно повторяя циклы записи (подробнее см. в $\left[{ }^{2-5}\right]$ ).

Любопытно отметить, что в принципе пространственно-временная голография осуществима и на обычных фотоматериалах. В теоретических работах $\left[14,{ }^{15}\right]$ предложен метод, в котором фиксирование спектрального состава объектного импульса достигается использованием специальной опорной волны с изменяющейся в глубь голограммы несущей частотой.

Задача теоретического описания голографического процесса в высокоселективной фотохромной среде в общем случае крайне сложна. Так, из-за сильной нелинейности среды (диэлектрическая проницаемость изменяется под действием света) имеет место обратное воздействие образующейся голограммы на записываемые поля, что особенно существенно в случае объемных голограмм. Далее, строгое вычисление восстановленной волны требует решения уравнений Максвелла для поля в пространственно и спектрально неоднородной среде, что при требуемой общности неосуществимо. Поэтому мы ограничимся приближением малого контраста записи, а распространение поля через голограмму рассмотрим, как обычно в задачах голографии и когерентной оптики (см. напр., $\left.\left[{ }^{16}\right]\right)$, в духе приближения Кирхгофа. Подробный анализ, проведенный для случая плоской голограммы, приведен в следующем разделе. 


\section{2. Голография нестационарного поля в приближении плоской голограммы}

Пусть высокоселективный фотохромный элемент в виде пластинки толщиной $d$ находится в плоскости $z=0$ и имеет поперечные размеры $2 x_{\max } \times 2 y_{\max }$. Предположим, что от объекта в сторону пластинки распространяется световой импульс (рис. 1), описываемый скалярным (аналитическим) сигналом $\mathrm{S}(\vec{r}, t)$, который в местоположении пластинки имеет вид

$$
\mathrm{S}(\vec{r}, t)=s\left(x, y, t-\frac{z}{c}\right) \cdot e^{i \omega_{0}\left(t-\frac{z}{c}\right)} .
$$

Комплексная амплитуда $s$ по сравнению с экспонентой слабо зависит от своих аргументов, причем ее зависимость от времени может отражать как амплитудную, так и частотную (около средней несущей частоты $\left.\omega_{0}\right)$ модуляции объектов-источников, а также их движение. Считаем выполненным обычное в оптике условие узкополосности: $\Delta \omega_{\mathrm{s}} \ll \omega_{0}$, где $\Delta \omega_{\mathrm{S}}$ - ширина спектра сигнала. Запись сигнала в форме (1) предполагает, что вид волн практически не изменяется при прохождении ими расстояния порядка $d$. Это означает, что либо угловые расстояния $\Theta_{z}$ источников от оси $z$ на объектной сцене, либо толщина пластинки должны быть достаточно малы: $\Theta_{z}^{2} \cdot d \leqslant \lambda$, где длина волны $\lambda=$ $=2 \pi c / \omega_{0}$. Для определенности выберем временную шкалу так, что в момент $t=0$ передний фронт импульса коснется пластинки, а к моменту $t_{\mathrm{S}}$ задний фронт покинет ее (когда затухание импульса асимптотическое, тогда выберем $t_{\mathrm{S}}$ так, что при $t \geqslant t_{\mathrm{S}}$ сигнал практически равен нулю).

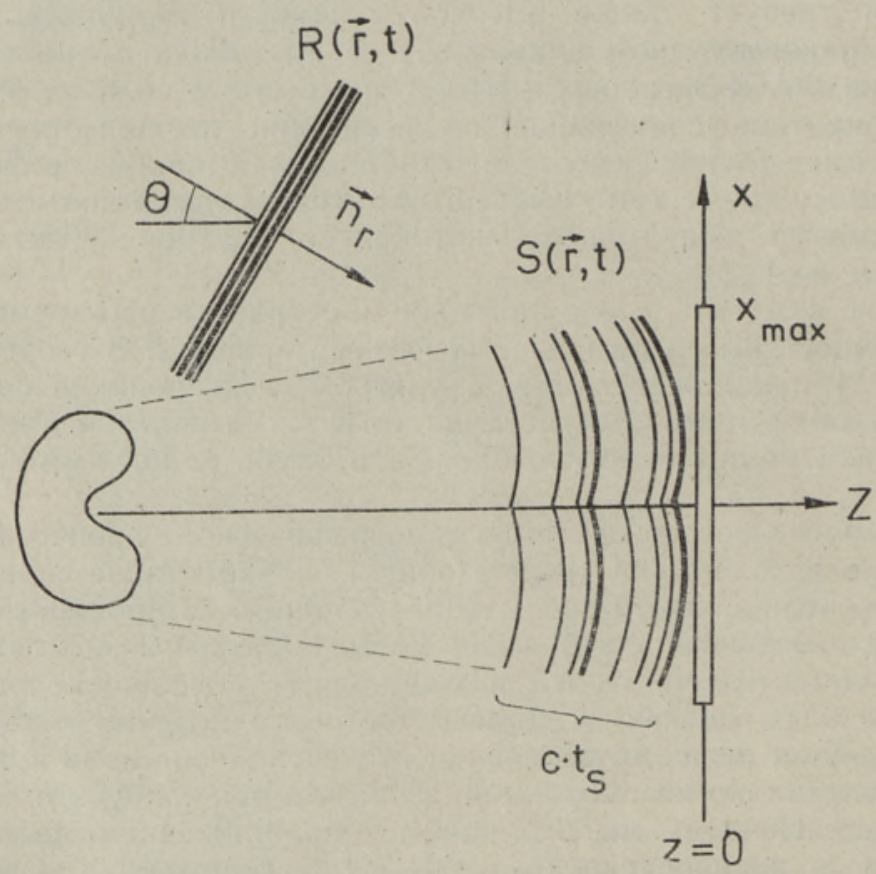

Рис. 1. Схема записи пространственно-временной голограммы. Излученный объектной сценой сигнальный импульс $\mathrm{S}(\vec{r}, t)$ в виде цуга волн изображен в момент времени $t=0$. При $0>t \geqslant t_{\mathrm{S}}$ значение $\mathrm{S}$ в плоскости $z=0$ практически равно нулю. Oпорный импульс $R(r, t)$ изображен для случая его задержки $t_{\mathrm{R}}>t_{\mathrm{s}}$. 
Опорный сигнал $\mathrm{R}(\vec{r}, t)$ выберем в видё импульса плоской волны, падающей на пластинку под углом $\Theta$ (рнс. 1)

$$
\mathrm{R}(\vec{r}, t)=\mathrm{R}_{0} \delta\left(t-\frac{\vec{n}_{\mathrm{R}} \cdot \vec{r}}{c}-t_{\mathrm{R}}\right) \cdot e^{i \omega_{0}\left(t-\frac{\vec{n}_{\mathrm{R}} \cdot \vec{r}}{r}-t_{\mathrm{R}}\right)}
$$

где $\overrightarrow{n_{R}}=(-\sin \Theta, 0, \cos \Theta)$ - единичный вектор в направлении распространения волны, $t_{\mathrm{R}}$ - момент прохождения точки начала координат пиком импульса с амплитудой $\mathrm{R}_{0}$. Предположение $\delta$-образности здесь сводится к требованию, что ширина спектра опорной волны $\Delta \omega_{R}$ больше ширины спектра сигнала и сам спектр практически постоянный в области спектра сигнала.

Фотохромная среда пусть характеризуется диэлектрической проницаемостью

$$
\varepsilon(\vec{r}, \omega)=\varepsilon_{0}+\frac{\sigma \cdot c}{2 \pi \omega} \int \frac{g(\vec{r}, \Omega) d \Omega}{\Omega-\omega+\frac{i}{T_{2}}}
$$

Здесь второй член обусловлен присутствием в среде поглощающих центров с неоднородным распределением частот перехода около $\omega_{0}$ и соответствует полосе поглощения, образовавшейся из бесфононных линий шириной $T_{2}{ }^{-1}$ примесных молекул с интегральным сечением поглощения б. Произведение $g(r, \Omega) d \Omega$ дает число центров в единице объема (до или после записи голограммы), которые имеют частоту бесфононной линии в интервале $\Omega ; \Omega+d \Omega$. Наличием фононных крыльев линий пренебрежем. $\varepsilon_{0}-$ обусловленная другими внутримолекулярными вибронными переходами и средой часть проницаемости, для простоты в дальнейшем $\varepsilon_{0}=1$. Предположим еще, что характерная ширина $\Delta \omega_{g}$ распределения $g$ больше ширины $\Delta \omega_{\mathrm{S}}$ и $\Delta \omega_{\mathrm{R}}$, а время фазовой релаксации резонансного перехода $T_{2}$ намного превышает суммарную длительность события сигнал плюс опорный импульс. Тогда, используя символическое равенство $\lim _{T_{2} \rightarrow \infty}\left(x+\frac{i}{T_{2}}\right)^{-1}=-i л \delta(x)+P \frac{1}{x}$, где $P-$ символ главного значения, вместо (3) можно написать

$$
\varepsilon(\vec{r}, \omega)=1-\frac{\sigma \cdot c}{2 \omega}[i g(\vec{r}, \omega)-\hat{H}\{g(\vec{r}, \omega)\}],
$$

где $\hat{H}\{g(\omega)\}$ - гильберт-образ функции $g(\omega)$.

Существенные соотношения частотно-временных характеристик модели изображены на рис. $2, \AA$. В случае низкотемпературных матриц с примесью органических красителей они выполняются для субнаносекундных сигнальных импульсов.

Теперь можем приступить к анализу голографического процесса по известной схеме, т. е. вычислим: 1) действующее при записи суммарное поле, 2) образовавшиеся в результате экспозиции изменения в диэлектрической проницаемости среды и 3) поле на выходе голограммы при считывании. Однако сразу же оговорим различия по сравнению с обычной голографией стационарных пространственных сцен. Во-первых, при записи необязательно, и как мы увидим в дальнейшем, даже нежелательно одновременное воздействие сигнального и опорного полей на голограмму. Во-вторых, требование когерентности сигнального и опорного полей встанет лишь в случае, если запись осуществляется говтор- 
A

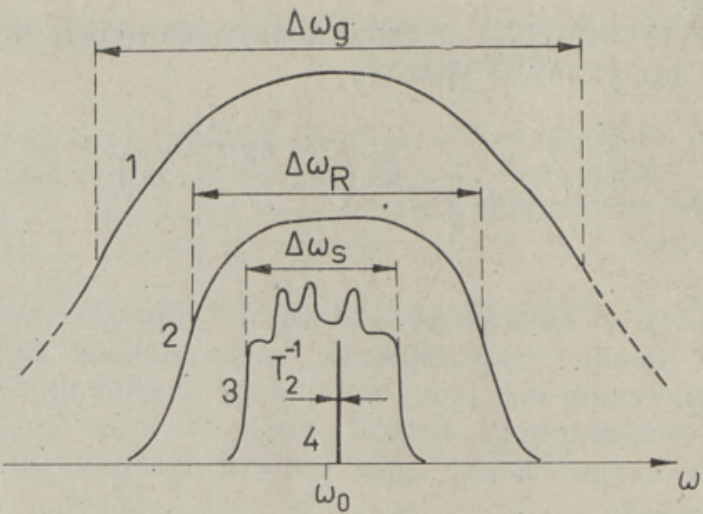

5

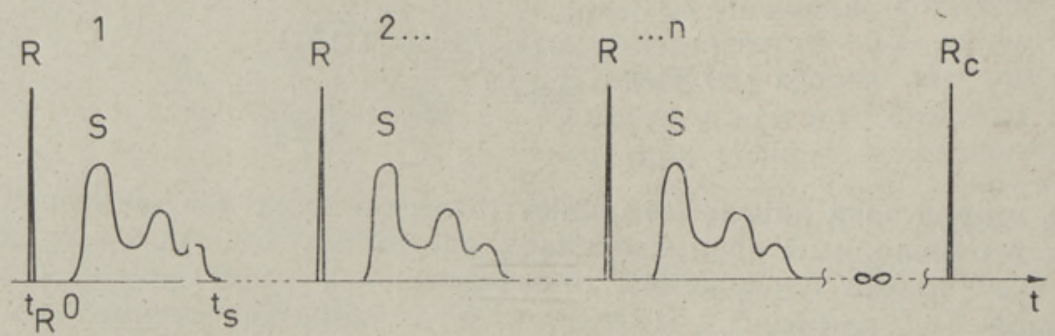

Рис. 2. $A$ - соотношение ширин спектров неоднородной полосы поглощения $(1)$, опорного импульса (2), сигнального импульса (3) и однородной бесфононной лннии центра (4). Б - временная последовательность подачи импульсов на голограмму; запись осуществляется одной или $n$ парами импульсов R и S, причем интервалы между парами превышают время фазовой релаксации $T_{2}\left(\sim 10^{-8} \mathrm{c}\right)$; после записи через произвольный промежуток времени (порядка нескольких часов, а может быть и месяцев) подается считырающий импульс $\mathrm{Re}$.

ными экспозициями, т. е. сигнальный и опорный импульсы многократно, через промежутки времени, превышающие $T_{2}$, воздействуют на голограмму (см. рис. 2, Б). В-третьих, анализ проведем, разложив поля на монохроматические составляющие, так как фоточувствительность среды спектрально селективна. Из-за последнего обстоятельства, которое, собственно, и позволяет охватить временную координату, голограмма будет всегда амплитудно-фазовой.

Поле опорного и сигнального импульсов на частоте $\omega$ вблизи $\omega_{0}$ в местоположении пластинки вычисляется преобразованием Фурье суммы (1) и (2)

$$
\bar{E}(\vec{r}, \omega)=\mathrm{R}_{0} e^{i \omega\left(x \sin \Theta-z \cos \theta-t_{\mathrm{R}}\right)}+\bar{s}\left(x, y, \omega-\omega_{0}\right) e^{-i \omega z},
$$

где черточка над символом обозначает фурье-образ, для упрощения записи $c=1$. Умножив (4) на комплексно-сопряженную величину, получим спектральную интенсивность на частоте $\omega$

$$
\begin{gathered}
I(x, y, \omega)=\bar{E} \bar{E}^{*}=\mathrm{R}_{0}^{2}+\left|\bar{s}\left(x, y, \omega-\omega_{0}\right)\right|^{2}+ \\
+\mathrm{R}_{0} \bar{s}\left(x, y, \omega-\omega_{0}\right) e^{-i \omega\left(x \Theta-t_{\mathrm{R}}\right)}+\mathrm{R}_{0} \bar{s}^{*}\left(x, y, \omega-\omega_{0}\right) e^{i \omega\left(x \Theta-t_{\mathrm{R}}\right)} .
\end{gathered}
$$

В (5) учтено, что $\Theta$ незначительно превышает малые углы $\Theta_{z}$, т. е. $\sin \Theta \approx \Theta$. В этом приближении (тонкой голограммы) $1-\cos \Theta \approx 0$, и зависимость от $z$ исчезает в экспонентах выражения (5). 
Рассмотрим теперь образование голограммы в пластинке благодаря процессам, при которых центры поглощения с вероятностью, пропорциональной $I(x, y, \omega)$, либо уничтожаются, либо приобретают новую частоту перехода вне полосы частот $I(x, y, \omega)$. Предположим, что в соответствии с условиями эксперимента 1) до записи плотность центров постоянна по всей пластинке и в полосе частот $I(x, y, \omega): g(\vec{r}, \Omega)=g_{0}$; 2 ) пластинка оптически толстая: $\sigma g_{0} d \gg 1$. Ограничимся линейным участком процесса прожигания голограммы, т. е. считаем изменения $g$ до: статочно малыми, так что отсутствует обратное влияние изменений

$\varepsilon(\vec{r}, \omega)$ на поле при записи (это обратное влияние отсутствует и в случае обычных фотоматериалов). Тогда плотность єентров после записи выражается

$$
\begin{gathered}
g(\omega, \vec{r})=g_{0}\left[1-\sigma n \eta I(x, y, \omega) e^{-g_{0} \sigma z}\right], \\
\sigma n \eta I \ll 1,
\end{gathered}
$$

где $n-$ число использованных для экспозиции пар сигнального и опорного импульсов, $\eta$ - вероятность превращения возбужденного светом центра, если $\mathrm{R}_{0}$ и $s$ определены так, чтобы $I$ имела размерность числа фотонов на единицу частоты и площади; экспоненциальный множитель учитывает ослабление поля в глубь пластинки.

В дальнейшем удобно использовать хорошо известный в когерентной оптике подход, при котором свойства голограммы-транспаранта описываются комплексным коэффициентом пропускания $K(x, y)$ считывающей волны. В случае примесного поглощения второй член в $\left(3^{\prime}\right)$ мал по сравнеиию с единицей и для $K(x, y)$ при частоте $\omega$ получим из $\left(3^{\prime}\right)$

$$
K(x, y, \omega)=e^{-i \omega d} \cdot e^{\frac{1}{2} \sigma \int_{0}^{d}[g(\omega, \vec{r})+i \hat{H}\{g(\vec{r}, \omega)\}] d z} .
$$

где первый множитель описывает тривиальное изменение фазы волны за счет прохождения пластинки конечной толщины $d$, который мы в дальнейшем опускаем. Из (6) и (7) получим окончательно для коэффициента пропускания голограммы после экспозиции при условии $\left(6^{\prime}\right)$, позволяющего ограничиться первым членом в разложении экспоненты

$$
K(x, y, \omega)=e^{-\frac{1}{2} g_{0} \sigma d}\left[1+\frac{1}{2} x(1+i \hat{H})\{I(x, y, \omega)\}\right],
$$

где коэффициент прожигания $x=\sigma n \eta$, а оператор $(1+i \hat{H})$ введен для сокращения записи в дальнейшем: $\quad(1+i \hat{H})\{f(\omega)\}=f(\omega)+i \hat{H}\{f(\omega)\}$.

Перейдем теперь к рассмотрению восстановления записанного поля при считывании голограммы. Нусть в направлении опорного импульса $\vec{n}_{\mathrm{R}}=(-\sin \Theta, 0, \cos \Theta) \approx(-\Theta, 0,1)$ на пластинку-голограмму падает плоская монохроматическая волна с частотой $\omega_{0}$. Тогда непосредственно за пластинкой $(z \rightarrow+0)$ возникает волна

$$
\bar{E}_{\omega_{0}}^{o u t}=e^{i \omega_{0}(t+\Theta x-z)} \cdot K\left(x, y, \omega_{0}\right),
$$

которая, учитывая (5), (8) и производя замену переменных $\omega=\omega_{0}+\Omega$ в (5), выражается:

$$
\vec{E}_{\omega_{0}}^{o u t}=e^{-\frac{1}{2} \sigma g_{0} d}\left[1+\frac{1}{2} x(1+i \hat{H})\left\{\mathrm{R}_{0}^{2}+|\bar{s}(x, y, \Omega)|^{2}\right\}\right] e^{i \omega_{0}(t+\theta x-z)}+
$$




$$
\begin{gathered}
+e^{-\frac{1}{2} \sigma g_{0} d}\left[\frac{1}{2} x \mathrm{R}_{0}(1+i \hat{H})\left\{\bar{s}(x, y, \Omega) e^{-i \Omega\left(\Theta x-t_{\mathrm{R}}\right)}\right\}\right] e^{i \omega_{0}\left(t+t_{\mathrm{R}}-z\right)}+ \\
+e^{-\frac{1}{2} \sigma g_{0} d}\left[\frac{1}{2} x \mathrm{R}_{0}(1+i \hat{H})\left\{\bar{s}^{*}(x, y, \Omega) e^{i \Omega\left(\Theta x-t_{\mathrm{R}}\right)}\right\}\right] \cdot e^{i \omega_{0}\left(t-t_{\mathrm{R}}+2 \Theta x-z\right)}, \\
\Omega=0, \quad z=+0 .
\end{gathered}
$$

Видим, что как и в случае обычной плоской голограммы, на выходе возникают три волны соответственно строкам выражения (10). Первая описывает распространяющуюся в направлении $\vec{n}_{\mathrm{R}}$ искаженную считывающую волну; вторая распространяется в направлении $z$ и дает мнимое изображение объектной сцены, в данном случае как бы наблюдаемой через узкополосный фильтр на частоте $\omega_{0}$; третья распространяется в направлении $(-2 \Theta, 0,1)$ и дает действительное изображение той же самой сцены.

Пусть теперь в направлении $\overrightarrow{n_{R}}$ на пластинку падает совокупность плоских монохроматических волн с частотами, равномерно покрывающими полосу $I(x, y, \Omega)$. Тогда каждая волна на выходе преобразуется согласно $(10)$, а результирующая волна получается суммированием (интегрнрованием) по всем частотам $\Omega$. Пусть совокупность падающих волн есть фурье-разложение считывающего импульса с характеристиками, аналогичными опорному, который проходит начало координат в некоторый момент времени $t=0$. Тогда выходящее поле $E^{\text {out }}(x, y, t)$ в плоскссти $(x, y)$ дается интегрированием выражения $(10)$ с фазовым множителем $e^{i \Omega(t+\Theta x)}$, т. е. обратнып преобразованием Фурье. Учитывая, что обратный фурье-образ выражения $(1+i \hat{H})\{\bar{f}(\Omega)\}$ есть $2 Y(t) f(t)$, где $Y(t)$ - единичная ступенька Хэвисайда, получим окончательно

$$
\begin{gathered}
e^{\frac{1}{2} \sigma g_{0} d} \cdot E^{o u t}(x, y, t)=\left[\mathrm{R}_{0}\left(1+\frac{1}{2} x \mathrm{R}_{0}^{2}\right) \delta(t+\Theta x)+\right. \\
\left.+\mathrm{R}_{0} x Y(t+\Theta x) \int s(x, y, \tau) s^{*}(x ; y,-t-\Theta x+\tau) d \tau\right] e^{i \omega_{0}(t+x \Theta)}+ \\
+\mathrm{R}_{0}^{2} x Y(t+\Theta x) s\left(x, y, t+t_{\mathrm{R}}\right) \cdot e^{i \omega_{0}\left(t+t_{\mathrm{R}}\right)}+ \\
+\mathrm{R}_{0}^{2} x Y(t+\Theta x) s^{*}\left(x, y ; t_{\mathrm{R}}-t-2 \Theta x\right) \cdot e^{i \omega_{0}\left(t-t_{\mathrm{R}}+2 \Theta x\right)}
\end{gathered}
$$

Видим, что выходящее поле как импульсный отклик голограммы подчиняется требованию причинности: функции $Y$, имеющие при $z \geqslant 0$ и точной записи аргумент $(c t+x \sin \Theta-z \cos \Theta)$, обеспечивают отсутствие поля в любой точке за голограммой до момента, когда эту точку пройдет считывающий импульс или его воображаемый передний фронт в точках, до которых сам импульс ввиду его ограниченной апертуры не доходит. Выражение (11) описывает три разделяющиеся на некотором расстоянии импульса волн (рис. 3). Первое слагаемое с амплитудным множителем в квадратных скобках описывает прошедший считывающий импульс с «хвостом», определенным функцией автокорреляции сигнала. При условии $t_{\mathrm{R}} \leqslant-\left|\Theta x_{\max }\right|$, где $x_{\max }-$ координата края голограммы, т. е. если при записи опорный импульс подавался на пластинку до сигнала, во втором члене функция $Y(t+\Theta x)$ окажется лишней. Следовательно, второй член в этом случае описывает восстановленное сигнальное поле (сравни с (1)), выходящее из голограммы с задержкой $t_{\mathrm{R}}$ относительно считывающего импульса. Наблюдатель вблизи оси $z$ воспринимает это поле как мнимое изображение записанной сцены. Важно 


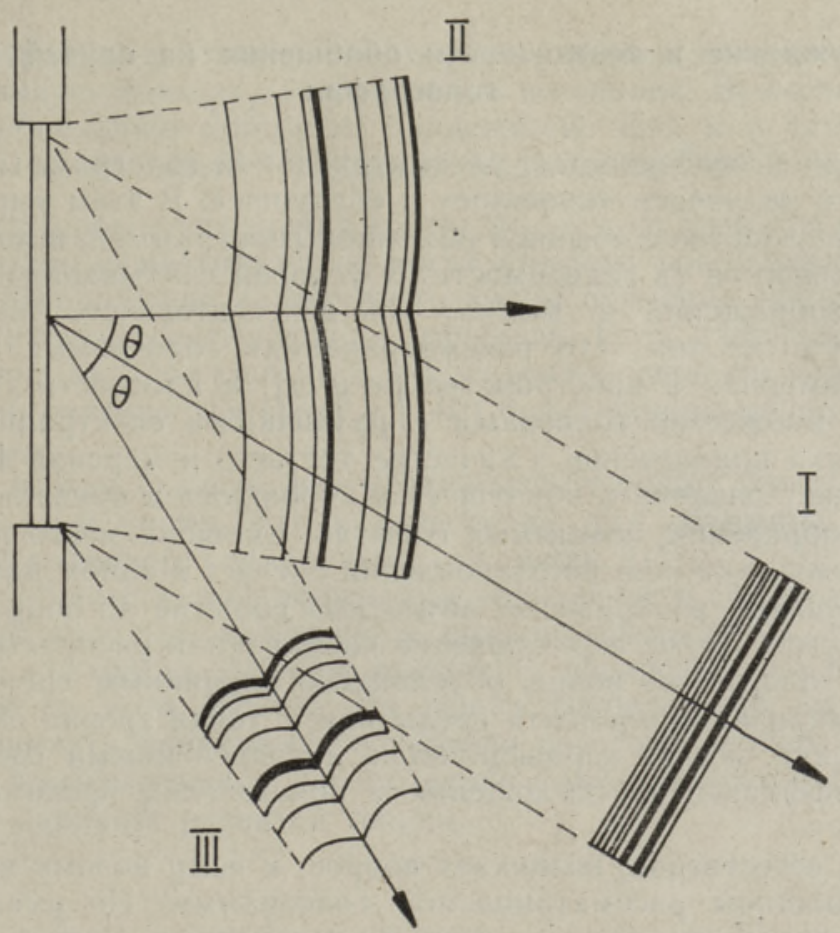

Рис. 3. Выходящие из голограммы импульсы при считывании. Римскими цифрами показано соответствие слагаемым выражения (11).

подчеркнуть, что имеет место полное восстановление объектной сцены во всей ее временной зависимости, включая возможную частотную модуляцию излучения источников. Если условие $t_{\mathrm{R}} \leqslant-\left|\Theta x_{\max }\right|$ не выполнено, происходит искажение сигнального импульса из-за обрезающего действия функции $Y$. Например, если сигнал состоит из двух импульсов, из которых задний достигает пластинки после опорного импульса, то в восстановленном в направлении $z$ поле второй импульс отсутствует. Если $t_{\mathrm{R}} \geqslant\left|\Theta x_{\max }\right|+t_{\mathrm{s}}$, т. е. когда опорный импульс подается после прохождения сигнала через пластинку, то первый член в (11) обращается в нуль и мнимого изображения в направлении $z$ не возникает.

Суть третьего члена в (11) становится очевидным, если предположить, что считывающий импульс подан в направлении $-\vec{n}$. Тогда третий член пишется (для $z=-0$ ) в виде

$$
\mathrm{R}_{0}^{2} x Y(t-\Theta x+z)\left[s\left(\vec{r}, t_{\mathrm{R}}-t-z\right) \mathrm{e}^{i \omega_{0}\left(t_{R}-t-z\right)}\right]^{*} .
$$

Легко заметить, что при условии необрезанного восстановления мнимого изображения $t_{\mathrm{R}} \leqslant-\left|\Theta x_{\max }\right|$ этот член равен нулю. Наоборот, при условии отсутствия мнимого изображения $Y(t-\Theta x+z)$ окажется лишним, и рассматриваемое выражение описывает обращенное во времени распространение сигнального поля *, т. е. в местоположении объекта записанная сцена разыгрывается в обратной временной последовательности. Поскольку плоская голограмма преобразовывает считывающую волну независимо от ее направления, сказанное остается в силе и в случае первоначального направления $\vec{n}_{\mathrm{R}}$ считывающего импульса, т. е. третий член в (11) описывает образование действительного изображения в направлении $(-\sin \Theta, 0, \cos 2 \Theta)$ с обращенным ходом времени (рис. 3).

* Оставшаяся комплексная сопряженность в $\left(11^{\prime}\right)$ не имеет физического значения. 


\section{3. Обсуждение и возможности обобщения на случай объемной голографии}

Замєчательной особенностью рассматриваемой голограммы является ее способность различать «прошлое» и «будущее». В этом выражается определенное сходство с обычной объемной голограммой, в которой также восстанавливается (в зависимости от условий считывания) только одно из двух изображений - мнимое или действительное. Аналогия здесь основывается на том, что рассматриваемая голограмма по существу также трехмерная в пространстве $[x, y, \omega]:$ в плоскость $(x, y)$ как бы наложено множество голограмм с различными спектральными частотами. Однако привлечение в качестве третьего измерения спектральной оси позволяет различить «будущее» и «прошлое» в буквальном смысле: мнимое изображение объектных событий, имевших место после подачи опорного импульса, при восстановлении следует в одном направлении, а (действительное) изображение объектных событий до опорного импульса - в другом. Такое переключение направления распространения рассеянной в голограмме волны обусловлено причинным временным поведением поляризации элемснта среды или, с точки зрения спектрального представления, всегда справедливыми дисперсионными соотношениями между коэффициентом поглощения и показателем преломления среды (см. $\left.\left(3^{\prime}\right)\right)$.

Теперь, естественно, возникает вопрос, к чему новому приведет увеличение толщины рассматриваемой голограммы. Не имея здесь возможности развивать полную теорию, рассмотрим случай сферической сигнальной волны, испущенной элементом объекта в точке $\vec{R}_{0}$ :

$$
\mathrm{S}(\vec{r}, t)=|\varrho|^{-1} \cdot s(t-\vec{n} \underline{\varrho}) \cdot e^{i \omega_{0}(t-\vec{n} \vec{\rho})}
$$

где $|\vec{\varrho}|=\left|\vec{r}-\vec{R}_{0}\right|-$ расстояние от источника до точки $\vec{r}$ в голограмме, $\vec{n}$ - единичный вектор в направлении $\vec{\varrho} ; \vec{n} \vec{\varrho}=|\vec{\varrho}|$. На основе вычислений, проведенных в разделе 2, можем сразу выписать (без несущественных констант) обратный фурье-образ пространственно-спектральной голограммы от (12) и (2) в среде, для определенности заполняющей правое от плоскости $z=0$ полупространство

$$
\begin{gathered}
Y(t) e^{i \omega_{0} t}\left[\delta(t)+|\vec{\varrho}|^{-2} \int s(\tau) s^{*}(\tau-t) d \tau+\right. \\
+|\overrightarrow{\mathrm{Q}}|^{-1} e^{i \omega_{0}\left(\vec{n}_{\mathrm{R}} \vec{r}-\vec{n} \vec{\rho}+t_{\mathrm{R}}\right)} \int s\left(t-\overrightarrow{n_{\mathrm{Q}}}-\tau\right) \delta\left(\tau+\vec{n}_{\mathrm{R}} \vec{r}+t_{\mathrm{R}}\right) d \tau+ \\
\left.+|\overrightarrow{\mathrm{Q}}|^{-1} e^{i \omega_{0}\left(\vec{n}_{\mathrm{R}} \vec{r}-\vec{n} \vec{\rho}+t_{\mathrm{R}}\right)} \int s^{*}\left(-t-\overrightarrow{n_{\mathrm{Q}}}+\tau\right) \delta\left(\tau-\vec{n}_{\mathrm{R}} \vec{r}-t_{\mathrm{R}}\right) d \tau\right] .
\end{gathered}
$$

Выражѐние (13) есть не что иное, как отклик (затухание поляризации) элемента среды, если на этот элемент подать считывающий импульс. Для вычисления восстановленной волны надо взять свертку (13) со считывающим импульсом ** (2). Для двух последних членов, описывающих волны с отличающимися от считывающего импульса направлениями распространения, получим

$$
\begin{gathered}
|\varrho|^{-1} Y\left(t-\overrightarrow{n_{\mathrm{R}}} \vec{r}\right) s\left(t-\vec{n} \overrightarrow{\mathrm{Q}}+t_{\mathrm{R}}\right) \cdot e^{i \omega_{0}\left(t-\vec{n} \vec{\rho}+t_{\mathrm{R}}\right)} \\
|\mathrm{Q}|^{-1} Y\left(t-\overrightarrow{n_{\mathrm{R}} r}\right) s^{*}\left(t_{\mathrm{R}}-t-\overrightarrow{n_{\mathrm{Q}}}+2 \overrightarrow{n_{\mathrm{R}} r}\right) e^{i \omega_{0}\left(t-2 \overrightarrow{n_{\mathrm{R}}} \vec{r} \vec{n} \vec{\rho}-t_{\mathrm{R}}\right)} .
\end{gathered}
$$

При считывании, как и раньше, $t_{\mathrm{R}}=0$. 
(14a) представляет восстановленную расходящуюся сферическую волну, соответствующую мнимому изображению источника. Временная зависимость в изображении повторяет записанную. Как и в случае тонкой голограммы имеет место обрезание «прошлого» из-за причинности.

В (14б) вектор распространения волны $2 \vec{n}_{\mathrm{R}}-\vec{n}$ не является единичным, т. е. такие волны существовать не могут. Поэтому восстановление действительного изображения (с обратным ходом времени) требует обращения в пространстве считывающего импульса. Если направление считывающей волны не параллельно с направлением опорной волны, то векторы распространения для (14a) и (14б) будут одновременно отличными от единичного.

В итоге можно сказать, что увеличение толщины голограммы приведет ни к чему иному, как к известному для объемных голограмм ограничению волнового синхронизма (см., напр., $\left[{ }^{16}\right]$ ).

Сложнее обстоит дело с эффектами нелинейности среды, так как при значительной толщине голограммы вышеиспользованное предположение слабого контраста (малого изменения распределения центров) не выполняется во всем объеме. Из общих соображений вытекает, что должны возникать временные повторения восстановленных сигнальных импульсов. Количественное рассмотрение таких эффектов возможно в нелинейном варианте развитой теории.

\section{Заключение}

В данной работе теория обычного голографического процесса обобщена на случай фоточувствительных материалов, способных фиксировать не только пространственное распределение интенсивности поля, но и его спектральный состав. Предложенный и экспериментально реализованный в $\left[{ }^{1-5}\right]$ метод голографической записи в средах с фотохимически активными примесными центрами поглощения позволяет восстановить изображение объекта во всей его временной зависимости в наносекундной об̆ласти. По существу пространственно-временная голография решает проблему истинного восстановления событий, если иметь в виду, что известный в кинематографни способ покадровой съемки не дает возможности в прямом смысле восстановить движение в изображении сцены.

Авторы выражают искреннюю благодарность К. К. Ребане, а также Р. Каарли за полезные обсуждения, стимулировавшие завершение данной работы.

\section{ЛИ Т Е РА Т У РА}

1. Ребане А. К., Каарли Р. К., Саари П. М. Опт. и спектр., 55, вып. 3, 405-407 (1983).

2. Ребане А. К., Каарли Р. К., Саари П. М. Письма в ЖЭТФ, 38, вып. 7, 320-323 (1983).

3. Rebane, A., Kaarli, R., Saari, P., Anijalg, A., Timpmann, K. Opt. Commun., 47, № 3, 173-176 (1983).

4. Rebane, A., Kaarli, R. Chem. Phys. Lett., 101, № 3, 317-319 (1983).

5. Ребане А. К., Каарли Р. К. Изв. АН СССР. Сер. физ.. (в гечатн).

6. Гороховский А. А., Каарли Р. К., Ребане Л. А. Пнсьма в ЖЭТФ, 20, вып. 7, $474-479$ (1974).

7. Kharlamov, B. M., Personov, R. I., Bykovskaya, L. A. Opt. Commun., 12, № 2, 191-193 (1974).

8. Ребане K. К. Элементарная теория колебательной структуры примесных центров кристаллов. М., «Наука», 1968.

9. Электромагнитное сверхизлучение. Қазань, АН СССР, 1975. 
10. Shtyrkov, E. I., Samartsev, V. V. Phys. status solidi (Q), 45, 647-655 (1978).

11. Денисюк Ю. Н. В кн.: Проблемы оптической голографии (под ред. Ю. Н. Денисюка). Л., «Наука», 1981, 7-27.

12. Зуйков В. А., Самарцев В. В., Усманов Р. Г. Письма в ЖЭТФ, 32, вып. 4, 293-297 (1980).

13. Mossberg, T. W. Opt. Lett., 7, № 2, 77-79 (1982).

14. Зубов В. А., Кузнечова Т. И. Квант. электроника, 13, № 1, 135-137 (1973).

15. Кузнецова Т. И. Тр. Физ. ин-та АН СССР, 84, 136-164 (1975).

16. Сороко П. М. Основы голографии и когерентной оптики, М., «Наука», 1971.

Институт физики

Академии наук Эстонской ССР
Поступила в редакцию 30/III 1984

\section{P. SAARI, A. REBANE}

\section{IMPULSSVALGUSVÄLJADE AJALIS-RUUMILINE HOLOGRAAFIA KORGSELEKTIIVSETES FOTOKROOMSETES KESKKONDADES}

Lähtudes varasematest eksperimentaalsetest tulemustest $\left[{ }^{1-5}\right]$, on esitatud muutuvatẻ objektide valgusvälja ajalis-ruumilise salvestamise ja taasesitamise holograafilise printsiibi teoreetiline käsitlus. Hologrammide salvestamine toimub objekti kiirguse ja tugiimpulsi koosmõjul kõrgselektiivsele fotokroomsele keskkonnale, mis fikseerib kiirguse sumnaarse peenstruktuurse intensiivsuse spektri. Hologrammi hilisem valgustamine üksiku ülilühikese impulsiga vôimaldab taasesitada objekti kiirguse nii esialgsel kui ka ajas ja ruumis pöoratud kujul.

\section{P. SAARI, A. REBANE}

\section{TIME-AND-SPACE-DOMAIN HOLOGRAPHY OF PULSED LIGHT FIELDS IN SPECTRALLY SELECTIVE PHOTOACTIVE MEDIUM}

A theoretical consideration of time-and-space-domain holography of ultrashort light pulses is presented which had been experimentally accomplished in $\left[^{1-5}\right]$. The storage is carried out by a coherent action of signal and reference pulses on a photoactive medium, which fixes their fine intensity spectrum structure. By illumination of the hologram with a single ultrashort read-out light pulse both an original and a timeand-space-reversed replica of the signal can be produced. 